\title{
COMENTARIO EDITORIAL:
}

\author{
Mortalidad materna en argentina
}

Resumen
Recientemente se ha publicado en Argentina una investigación multidimensional que aborda en forma integral "el camino de la
muerte materna" desde la dinámica de los factores familiares, personales y comunitarios en el marco de los cuales ocurre el
embarazo, pasando por la accesibilidad a los servicios de salud y la calidad de las prestaciones brindadas por el sistema de
salud a la mujer embarazada y durante el período puerperal, hasta la modalidad de notificación, codificación y registro de las salud a la mujer embertes maternas.

En este número de la revista EVIDENCIA hemos resumido y comentado en sendos artículos editoriales dos de los capítulos de este excelente trabajo: 1) estudio de casos y controles que investiga la asociación estadística entre factores vinculados al proceso de atención y la probabilidad de defunción materna; 2) investigación cualitativa que intenta reconstruir la historia y el camino recorrido por la mujer desde que percibió algún malestar hasta que falleció.

Los resultados del estudio de casos y controles sugieren que contar con profesionales capacitados/acreditados en los servicios de atención obstétrica y con los insumos necesarios para asistir una emergencia, podría ser una respuesta eficaz para contrarrestar la mortalidad materna.

Respecto de la investigación cualitativa, el modelo de las tres demoras (en buscar atención, en llegar al servicio de salud y en recibir el tratamiento adecuado dentro del servicio) habla por si mismo respecto de la catástrofe sanitaria que afecta a nuestra región.

\section{En el camino de la muerte materna sólo quedan palabras}

Se define muerte materna a la que le ocurre a una mujer mientras está embarazada o dentro de los cuarenta y dos días siguientes a la terminación del embarazo, independientemente de la duración y el sitio del mismo, debido a cualquier causa relacionada con o agravada por el embarazo mismo o su atención, pero no por causas accidentales o incidentales.

La mortalidad materna es un indicador directo que refleja múltiples dimensiones del estado de salud de una población y que indirectamente describe las condiciones sociales, económicas y culturales que actúan en una comunidad. Los países que han conseguido disminuirla han adoptado medidas globales de costo efectividad probada: planificación familiar para la prevención del embarazo no deseado, manejo apropiado del embarazo de alto riesgo, mejoramiento de los estándares y prácticas de personal calificado para el manejo del parto basado en la evidencia y provisión de abortos seguros; así como vigilancia de las muertes maternas para monitorear su evolución, distribución, determinantes y causas, y para evaluar el impacto de las intervenciones y realizar las acciones necesarias.La mayoría de las mujeres que mueren por causas asociadas a la maternidad son las más pobres, con menor nivel educativo, alta fecundidad y dificultades en el acceso a los servicios de salud'. Sin embargo, no alcanza el "abordaje de riesgo" para predecir qué mujeres tendrán complicaciones, siendo necesario asumir que cualquier complicación puede desarrollarse durante o después del embarazo en forma imprevista² ${ }^{2}$.

Los objetivos generales de la investigaciónde Ramos y col. ${ }^{3}$ que comentamos se plantearon en dos planos:

Un plano epidemiológico: a) realizar un diagnóstico de la situación de la mortalidad materna en seis provincias seleccionadas (Chaco, Formosa, Mendoza, San Juan, San Luis y Tucumán), b) analizar la dinámica de los determinantes sociales e institucionales de las muertes maternas y c) fortalecer las actividades del sistema de vigilancia epidemiológica de la mortalidad materna de los ministerios de salud nacional y provinciales.

Un plano político: a) desarrollar las capacidades de investigación de los equipos locales y $b$ ) formular recomendaciones para mejorar las políticas sanitarias y extrasectoriales en salud reproductiva orientadas a la reducción de la mortalidad materna.

En cuanto a los resultados, podemos decir que la falta de insumos, tanto de medicamentos e instrumentos como de sangre y personal técnico, observada en todos los niveles de atención, también significó importantes demoras. Aún luego de controlar el potencial efecto confundidor de las características biológicas y sociales de las mujeres, las variables relacionadas con la oferta de servicios (falta de disponibilidad de guardia obstétrica activa y de atención obstétrica esencial) mostraron fuerte asociación al riesgo de muerte materna.

Es interesante destacar la metodología empleada en la investi- gación cualitativa así como sus resultados. La Organización Mundial de la Salud (OMS) define las autopsias verbales (AV) como un proceso destinado a facilitar la identificación de muertes maternas donde la certificación médica es inadecuada 4 . Dado que tanto el informe estadístico de defunción como la historia clínica indicaban con claridad la causa de muerte, las autopsias verbales (AV) se utilizaron para reconstruir la dinámica de los factores familiares, personales y comunitarios que obstaculizaron el contacto oportuno de la mujer con el servicio de salud. El objetivo fue separar las verdaderas muertes maternas de aquellas que no lo fueron, mediante la reconstrucción de los eventos que rodearon al fallecimiento dentro de la comunidad.

La investigación cualitativa que comentamos informó que la situación de las mujeres que interrumpieron un embarazo se caracteriza por la falta de involucramiento de sus compañeros en el cuidado anticonceptivo y por el padecimiento de violencia por ellos ejercida. Esta asimetría opera como barrera clave para adoptar medidas anticonceptivas seguras y eficaces que les permitan evitar los embarazos no deseados y así, los abortos inseguros.

El riesgo que trae aparejado un aborto practicado en condiciones inseguras se potencia por la clandestinidad en la que se realiza y la condena social que lo rodea cuando se hace público. En este contexto social y legal, la búsqueda de atención médica ante las señales de alarma se demora. A pesar del silencio en el que se recluyeron las mujeres que decidieron interrumpir su embarazo, la posibilidad de contar con "otras personas significativas" agilizó el acceso y la obtención de atención médica.

El transporte hacia el centro de salud resultó un obstáculo importante que implicó demoras entre las mujeres residentes en zonas rurales, situación agravada por la falta de medios para la comunicación (teléfono). En otros casos, el trayecto desde el domicilio al servicio de salud se realizó en medios poco adecuados (bicicleta), aunque sin demoras.

En la recepción en el servicio y el tratamiento de la emergencia, tanto en la atención de las mujeres que presentaban complicaciones de abortos como de las que fallecieron por otras causas, se destacan situaciones de diagnóstico erróneo, tratamiento ambulatorio con calmantes y demoras en efectuar la derivación a un centro de mayor complejidad. En algunos casos, si bien la derivación fue realizada oportunamente, su ejecución se retrasó debido a la falta de medios de transporte del propio sistema de salud, lo que generó graves dilaciones. Si bien nos queda un gusto amargo, sólo nos queda felicitar a los autores de este magnífico trabajo, deseando que sus conclusiones nos ayuden a todos los ciudadanos -especialmente a quienes se encuentran en niveles de decisión política, a quienes estamos involucrados en los sistemas de salud y a todos los actores sociales con peso en el devenir de nuestra nación- a trabajar en forma coordinada para que, como han decidido ellos titular su informe, "cada muerte materna importe" y para que estas sean cada vez menos.

Nanci Giraudo [ Unidad de Medicina Familiar y Preventiva del Hospital Italiano de Buenos Aires. ] 


\section{Influencia de la organización de los servicios de salud en la mortalidad materna}

Tal como indican los Ramos y col. en su investigación ${ }^{3}$, se acepta que $15 \%$ de los nacimientos tiene una complicación potencialmente fatal que requiere asistencia de emergencia ${ }^{5}$. Además, estas complicaciones no pueden predecirse, por lo que se debe considerar que cada embarazo esta en condición de riesgo ${ }^{6}$.

En Argentina, la mayoría de las instituciones donde se asisten partos tiene menos de 1500 nacimientos anuales. Cabe destacar que no es necesariamente el número de nacimientos que asiste la institución lo que constituye el riesgo, sino que en general, dichas instituciones tienen menor acceso a anestesia (ni siquiera al llamado) de banco de sangre, de facilidades de traslado, de posibilidades de hacer cesárea, de neonatología, etc. Aproximadamente el $50 \%$ de las instituciones del subsector público donde se asisten partos, tiene estas carencias.

Cuando se piensa en sacar los partos de las instituciones que no reúnan las condiciones de seguridad básicas para la asistencia de nacimientos, se encuentra la resistencia de las autoridades y de la comunidad. Los pobladores quieren tener su "hospitalito" y quieren que sus hijos nazcan en su propio pueblo. Tampoco aceptan su traslado las madres multíparas para ser asistidas en una "maternidad segura" ya que tendrían que separarse de los otros hijos. En la investigación de Ramos y col. se muestra que los malos resultados en la salud materna son consecuencia de la ausencia de servicios y de la insuficiente calidad de cuidados que se brinda en los servicios existentes. La mala calidad se debe a la ausencia de recursos humanos y materiales, a tratamientos inadecuados (falta de competencia técnica) y a insuficiente intercambio de información.En la serie analizada, una de cada tres a cuatro muertes es por aborto. Pese a que actualmente existe una legislación que facilita el acceso a la planificación familiar, en el presente no hay motivos para suponer que esta proporción cambie. Este supuesto se basa en el hecho que las mujeres más pobres que ya están utilizando contracepción lo seguirán haciendo con mayor facilidad de acceso, mientras que quienes no la han utilizado hasta ahora, tienen que llegar a modificar pautas culturales (de la población y de los servicios) lo que va a demorar varios años.

Este tipo de estudios son importantes para pensar los factores de contexto y su impacto en la planificación de las respuestas sanitarias. Se destaca que los países que han logrado disminuir la mortalidad materna, aún con menos recursos en salud que la Argentina, lo hicieron no solo con medidas costo-efectivas, sino modificando factores de contexto.

Las autoridades de salud del área materno infantil, no siempre tienen la capacidad y legitimidad para liderar el planeamiento en sus provincias. No hay una política nacional de salud y las metas establecidas en los objetivos del desarrollo del milenio parecen poco realistas. Con frecuencia hay conflictos entre el nivel municipal y el provincial, lo que dificulta la planificación. Los ciclos políticos, como resultado de elecciones, son barreras a la continuidad del proceso de planificación (frecuentes cambios de responsables técnicos). Los cambios económicos y políticos (de la economía de mercado y del sistema estatal) han demostrado que la planificación no se adaptó a dichos cambios.

Lo primero que debe definirse es si se quiere un Plan de Salud o un Plan para el Sector Público. Si la opción es el Plan de Salud hay que:

- Poner un límite en el número, actividades y ubicación geográfica de las instituciones que atienden partos (nueva rehabilitación).

- Balancear adecuadamente los incentivos y los controles.

- Regular y acreditar a los profesionales.

- Desarrollar normas y estándares de cuidados.

- Desarrollar lineamientos para el funcionamiento institucional (redes de asistencia).

Para concluir, podemos decir que la mortalidad materna, al igual que la infantil, siempre disminuyen, el problema es a que velocidad lo hacen. El acceso a las instituciones pareciera estar asegurado ya que $98,6 \%$ de los partos son institucionales, pero, tal como lo demuestra la investigación de Ramos y col., no parecen ser muy seguras las instituciones.

Jorge Vinacur [ Médico Obstetra. Asesor de Estadísticas Vitales del Ministerio de Salud de la Nación. ]

\section{Referencias}

1. Langer, A. y Espinoza, H.: "Embarazo no deseado: impacto sobre la salud y la sociedad en América latina y el Caribe", en Nuevos desafíos de la responsabilidad política, Cuadernos del Foro de la Sociedad Civil en las Américas, año 4, № 5, Buenos Aires, CEDES-FLACSO-CELS, 2002.

2. Koblinsky, et al.: "Mother and more: a broader perspective on women's health", en M. Koblinsky, J. Timyan y J. Gay, The health of women. A global perspective, Boulder,Westview Press, Inc., 1993

3. Ramos S, Romero M, Karolinski A, Mercer R, Insua I, del Río Fortuna C editores. Para que cada muerte materna importe. Pag. 63-80. 2004, CEDES. ISBN: 950-9572-25-X.

4. Sloan, N. et al.: The etiology of maternal mortality in developing countries: what do verbal autopsies tell us?, Bulletin of the World Health Organization, 79, 2001, págs. 805-810.

5. WHO: "Reduction of Maternal Mortality", A joint WHO/UNFPA/UNICEF/World Bank Statement, Ginebra, 1999

6. Koonin LM, et al. Pregnancy related mortality surveillance. United States, 1987-1990. www.cdc.gov/mmwr/preview/mmwrhtml/00048923.htm)

\section{Acerca del tratamiento corto de neumonías en niños}

Recientemente salieron publicados en Evidencia dos trabajos paquistaníes con series muy grandes de pacientes sobre tratamientos cortos en neumonías leves de niños menores de cinco años. Uno con tres días de amoxicilina vs. cinco días ${ }^{1}$ y otro sobre cotrimoxazol vs. amoxicilina (dos veces por día) durante cinco días $^{2}$. El problema de ambos trabajos es su validez externa ya que en nuestro medio no hablamos de lo mismo cuando decimos neumonía. En los estudios mencionados se diagnosticó neumonia por la taquipnea. Si bien la OMS lo propone como método diagnostico, en Argentina (por lo menos por ahora) el diagnóstico de referencia es radiográgico. Si bien, la taquipnea es el mejor signo clínico (coeficiente de probabilidad positivo: 1,5-2,1 y coeficiente de probabili- dad negativo: $0,36-0,5)$ no alcanza por sí solo para diagnosticar o descartar neumonía ${ }^{3}$. Por ejemplo, en los menores de cinco años, muchas patologias respiratorias más frecuentes que la neumonia bacteriana se presentan con taquipnea y no requieren tratamiento antibiótico. Me refiero a la bronquiolitis y a las neumonías virales en los menores de dos años y a los primeros episodios de asma en los mayores de esta edad (los autores excluyeron a los que tenían tres o más broncoespasmos). Por lo expuesto no me sorprende que no hayan encontrado diferencias y que haya habido una falla de tratamiento cercana al $20 \%$ en ambas ramas de ambos trabajos. Probablemente se trate de los pacientes que sí necesitaban antibióticos y que ni con tres ni con cinco días haya sido suficiente. Con estas evidencias, considero que no habría que innovar en el método diagnóstico, ni la duración, ni en el esquema antibiótico del tratamiento empírico inicial.

Juan Pablo Mouesca [ Comite Editor Evidencia, Monte Grande. ]

\section{Referencias}

1. Pakistan Multicentre Amoxicilin Short Course therapy (MASCOT) pneumonia study group. Clinical fficacy of 3 day versus 5 days of oral amoxicillin for treatment of childhood pneumonia: a multicentre doble-blind trial. Lancet 2002; 360: 835-841.

2. CATCHUP study group. Eficacia clínica del cotrimoxazol versus amoxixilina, dos veces por día, para el tratamiento de neumonía: un ensayo clinico controlado, randomizado, en Paquistán. Arch Dis Child 2002; 86: 113-118.

3. Margolis P, Gadomski A. Does this pacient have pneumonia? JAMA, 1998; 279:308-313. 